Reducing relapse in psychosis through medication management.

Louise Doyle and Brian Keogh

Cite as: Doyle, L \& Keogh, B., Reducing relapse in psychosis through medication management., Mental Health Practice, 11, (9), 2008, p32 - 35

Louise Doyle RPN, BNS, RNT, MSc.

Lecturer in Psychiatric Nursing

School of Nursing and Midwifery Studies,

Trinity College Dublin,

24 D’Olier St.

Dublin 2

Ph: 0035318963102

Email: louise.doyle@tcd.ie

Brian Keogh RPN, BNS, MSc.

Lecturer in Psychiatric Nursing

School of Nursing and Midwifery Studies,

Trinity College Dublin,

24 D'Olier St,

Dublin 2

Ph: 0035318963109

Email keoghbj@tcd.ie 


\section{Reducing relapse in psychosis through medication management.}

\section{$\underline{\text { Introduction }}$}

Simonelli-Collen (2005) suggests that the concept of relapse is a complex, multidimensional phenomenon that is not uniformly defined in the research literature. However, it is commonly understood as the re-emergence or worsening of signs and symptoms in individuals previously treated for psychoses (Birchwood \& Spencer 2001). Relapse is often thought to be indistinguishable from setting to setting with a similar client experience. However it is remarkable for its divergence in quantity of symptoms experienced by individuals, acceleration and duration of onset. Successful relapse prevention offers the potential of lowering treatment resistant symptoms and decelerating the clients' possible passage into severe and enduring mental illness (Birchwood \& Spencer 2001). Relapse prevention consists of strategies designed to assist the client and significant others to monitor the client's condition over time to detect early warning signs of impending psychoses and the implementation of an individualised action plan to offset the development of a 'full blown' psychotic episode. In addition, strategies to help the person to recognise and manage precipitating factors that lead to relapse such as stress are developed using a biopsychosocial approach. The importance of relapse prevention can perhaps be summarised by Birchwood et al (1993) who argued that many clients feel trapped by their illnesses, which may make them more prone to depression. Subsequently they have a strong interest in learning to recognise and avoid the re emergence of symptoms and possible re hospitalisation (Birchwood et al 1993). Relapse prevention consists of multiple individualised approaches that are tailored to meet the diverse needs of clients who are attempting to manage their symptoms. Consequently the focus of the intervention is often psychosocial in nature. One strategy that has been 
consistently used in the management of symptoms is the use of psychotropic medications. This article focuses on medication management as one of the approaches used, in combination with others, to reduce relapse in clients with mental health problems.

\section{Adherence with psychotropic medications.}

The use of psychotropic medications remains one of the mainstays of psychiatric treatment however there is considerable evidence to suggest that many people do not take their medication as prescribed. It has been established that continuing on regular medication can significantly reduce relapse rates. Kisling (1994) has argued that if clients were completely adherent with their medication regimes, relapse rates would fall to about $15 \%$. Consequently there has been an effort to target 'non-compliance' among those who are prescribed these medications. There is therefore an onus on mental health nurses to play a pivotal role in this process.

In recent years there has been recognition that the term 'compliance' is value-laden and suggests an authoritative relationship between the client and the prescriber/carer (Marland and Sharkey 1999). Accordingly, the terminology has moved from 'compliance' to 'adherence' through to 'concordance' which suggests a partnership between client and prescriber and a more collaborative approach to care. These terms are still however used interchangeably in the available literature.

\section{Factors affecting adherence to psychotropic medications.}

The factors contributing to a client's decision not to take their medication as prescribed are numerous and diverse. These factors include: 
- Side-effect profile of prescribed medications: particularly side-effects such as extra-pyramidal side-effects which can be distressing and socially disfiguring but also effects such as weight gain and sexual dysfunction.

- Lack of insight into the illness: individuals may believe that they are not ill and therefore have no need to take medication.

- Clinical features of illness: e.g. paranoia or suspiciousness that may be present in certain illnesses.

- Secondary gain from illness: e.g. people who are manic may enjoy the feeling of elevation and may not want to take medication which they know will reduce this elevated mood.

- Lack of symptoms: this is particularly a problem for those who take medication prophylactically to prevent a re-occurrence of symptoms e.g. Lithium in bipolar disorder.

- Refusal to accept diagnosis: if a client does not believe they have a mental health problem they may be reluctant to take psychotropic medication.

- Beliefs about treatment: many people believe that medications should not have a role in treating mental health problems and this belief may affect their decision to take such medications.

- Fear of drug addiction: some clients may believe that all psychotropic medications are addictive and therefore may be reluctant to commence such treatment.

- Quality of relationship with the prescriber: paternalistic or collaborative? People who are involved in the decisions made about their medication regime are more likely to adhere to it. 
- Treatment setting: adherence to medication regimes is more likely in a hospital setting where there is supervision as opposed to a community/home setting.

- Dual diagnosis: co-morbidity of a mental health problem and a substance abuse problem increases the rate of non-adherence with medication regimes.

- Poor knowledge and understanding of medications.

- Polypharmacy/complex regimes leading to inability to understand medications.

Non-adherence with psychotropic medications can have a range of effects including an increase in relapse rate leading to an increased number of hospitalisations. This in turn may cause a loss of occupational/recreational opportunities and may cause a decrease in social functioning. Non-adherence may also increase the burden on carers and mental health services. It is therefore essential that interventions aimed at improving adherence rates are developed and implemented.

\section{Promoting medication management.}

A medication management approach must highlight the importance of interactions that encourage client participation, choice and autonomy. It must also aim to address the reasons behind non-adherence. The following are common interventions within a medication management approach:

Assessment of mental state: an initial assessment of the client's illness is important both prior to commencement of psychotropic medications and also during treatment to assess the medication efficacy. A baseline measurement of symptoms prior to administration of medications is also important to distinguish symptoms from side- 
effects. A scale such as the KGV symptom scale can be utilised (Krawiecka et al, 1977).

Assessment of side-effects: Assessing the severity of drug related side-effects is an important aspect of medication management. They may go undetected as clients may not report them and nurses may not ask about them (Bennett et al 1995). A number of neuroleptic side-effect rating scales exist which can help to provide an accurate picture of the extent of side-effects and how they impinge on clients' lives. However, one that can be easily incorporated into nursing practice is the Liverpool University neuroleptic side-effect rating scale (LUNSERS) (Day et al 1995). This 51-item scale asks the client to report the range of side-effects they have experienced and also elicits the stress which each side-effect causes the individual. Day et al (1995) identify that this scale is particularly useful as it can be administered by health care staff without special training, can be completed by acutely disturbed clients and only takes between 5-20 minutes to complete ensuring that it can be included in on-going nursing assessments.

Management of side-effects: many side-effects can be managed by downward titration of dosage, switching to other pharmacological therapies or by introducing other medications to alleviate side-effects. While traditionally these were primarily medical interventions, the introduction of nurse supplementary prescribing (NSP) in the UK in 2003 has ensured that mental health nurses have an expanding role in the area of prescribing medications. Aimed particularly for the management of chronic illnesses such as schizophrenia, NSP involves a voluntary partnership between the independent prescriber (the psychiatrist) and the supplementary prescriber (the nurse) who work together on a specific clinical management plan pertaining to a particular patient (Department of Health 2003). The supplementary nurse prescriber can, if 
appropriate, adjust the dose of medications and switch or stop medicines. This ensures that patients can have quicker and more efficient access to medications and can have a more expedient response to the occurrence of unwanted side-effects.

It is important to note that mental health nurses also have a key role in the symptomatic management of side-effects. Including non-medical interventions to alleviate common side-effects such as constipation, dry mouth and weight gain can be a crucial step in improving adherence with common psychotropic medications. These interventions could include dietary and lifestyle advice with a view to reducing/preventing constipation and weight gain and the use of an artificial saliva spray to help minimise the discomfort caused by dry mouth.

Compliance therapy (Kemp et al 1997): this is a cognitive-behavioural intervention that has adapted techniques from motivational interviewing and psychoeducation. The aims of the therapy are to develop an open dialogue about medication and to encourage discussion about the pros and cons of pharmacological treatment. It promotes a partnership approach between the client and the mental health professional to aid 'negotiating medication'. Clients have reported that they are often not consulted or included in the decision making process with regard to their medication regime (Gray et al 2005, Happell et al 2004). Excluding clients from the decision making process with regard to their medication is disempowering and is more likely to lead to non-adherence with a planned regime of psychotropic medication. There is therefore an absolute need to include the client in the decision making process and to listen to and respond to their concerns. This is not solely the remit of the prescribing medic or supplementary nurse prescriber but is particularly the role of the person who administers the medication; the mental health nurse. Having a positive therapeutic relationship with the person who prescribes and administers medication can be a 
crucial component to increasing adherence with medication. This relationship requires a therapeutic engagement which is a prerequisite when embarking on any mental health intervention. Historically, engaging with clients with severe mental illness in particular was an area embarked upon with some trepidation. It was considered that people with a diagnosis of schizophrenia were unable to collaborate in a working relationship because of their impaired mental state (Wolpe 1958). However, more contemporary thinking suggests that addressing concerns about the client's illness and treatment may lead to more satisfactory outcomes and improve their engagement with the mental health services. Compliance therapy involves working collaboratively with clients to address their beliefs and perceptions about medication, their ambivalence about change and their expectations about treatment outcomes. There is an emphasis on personal choice and responsibility and an interest in eliciting and exploring the client's concerns about their treatment.

Education is also a key component of compliance therapy and aims to provide information to clients about their illness and their medication to improve their understanding and increase adherence. It is reported that many clients are not provided with adequate information about the psychotropic medications prescribed to them (Gray et al 2005, Happell et al 2004). This may be a result of several different factors for example failure of the client to directly ask for information about their medications, a fear on the part of the health professional that giving full information about a medication may promote non-adherence or a reluctance on the part of the health professional to discuss certain side-effects with clients. Higgins et al (2005) cites the latter as an issue when looking at the side-effect of sexual dysfunction which may occur with some psychotropic medications, in particular antipsychotic and antidepressant medications. Addressing this issue Higgins et al (2005:444) suggest 
that "mental health nurses need to meet the challenge of informing service users with regard to these side effects, and supply education as to how some side effects may be diminished". However, while education is useful in improving people's knowledge about their medication, when used in isolation from the other components of compliance therapy, it does not increase adherence (Gray et al 2002). Finally, compliance therapy also involves a behavioural component which is concerned with incorporating the medication regime into the everyday routine of the client. Utilising this behavioural component, clients are encouraged to link taking their medication with everyday routine behaviours such as mealtimes, going to bed etc. It would appear that this intervention may be more suited to those clients whose non-adherence with medication is unintentional and due to forgetfulness rather than an intentional decision to stop taking their medication.

\section{Conclusion}

"Mental health nurses play a central role in helping patients manage their medication more effectively" (Gray et al 2002: 283). The introduction and increase in nurse prescribing ensures that mental health nurses have a greater than ever role to play in this important intervention. Understanding the factors that affect a person's decision to take their medication and working collaboratively with them in addressing these factors is crucial if clients are to benefit from the positive effects of medication while minimising adverse effects. In particular, mental health nurses through the use of techniques of medication management can play a positive role in preventing relapse for the individual client and thereby reducing the level of psychological and social disability resulting from this experience. 
References

Bennett J, Done J \& Hunt B (1995) Assessing the side-effects of antipsychotic drugs: a survey of CPN practice. Journal of Psychiatric and Mental Health Nursing, 2(3), $315-330$

Birchwood M, Mason R \& MacMillan F et al (1993) Depression, demoralisation and control over illness: a comparison of depressed and non-depressed patients with a chronic psychosis. Psychological Medicine, 23, 387-395

Birchwood M \& Spencer E (2001) Early intervention in psychotic relapse. Clinical Psychology Review, 21(8), 1211 - 1226.

Day JC, Wood G, Dewey M \& Bentallo RP (1995) A self-rating scale for measuring neuroleptic side-effects. Validation in a group of schizophrenic patients. British Journal of Psychiatry, 166, 650-653

Department of Health (2003) Supplementary Prescribing by Nurses and Pharmacists within the NHS in England: A guide for implementation. London: Department of Health.

Gray R, Wykes T \& Gournay K (2002) From compliance to concordance: a review of the literature on interventions to enhance compliance with antipsychotic medication. Journal of Psychiatric and Mental Health Nursing, 9, 277-284

Gray R, Rofail D, Allen J \& Newey T (2005) A survey of patient satisfaction with and subjectives experiences of treatment with antipsychotic medication. Journal of Advanced Nursing, 52(1), 31-37

Happell B, Manias E \& Roper C (2004) Wanting to be heard: mental health consumers' experiences of information about medication. International Journal of Mental Health Nursing, 13, 242-248

Higgins A, Barker P \& Begley CM (2005) Neuroleptic medication and sexuality: the forgotten aspect of education and care. Journal of Psychiatric and Mental Health Nursing, 12, 439-446

Kisling W (1994) Compliance, quality assurance and standards for relapse prevention in schizophrenia. Acta Psychiatrica Scandinavica, 89 (Supplement 382), 16-24

Kemp R, Hayward P \& David A (1997) Compliance Therapy Manual. London: Bethlem and Maudsley NHS Trust.

Krawiecka M, Goldberg D \& Vaughn M (1977) A standardised psychiatric assessment scale for rating chronic psychotic patients. Acta Psychiatrica Scandinavica, 55, 299-308 
Marland G \& Sharkey V (1999) Depot neuroleptics, schizophrenia and the role of the nurse: is practice evidence based? A review of the literature. Journal of Advanced Nursing, 30, 1255-1262

Simonelli-Collen M (2005) Relapse: A concept analysis. Nursing Forum, 40(1), 3 10

Wolpe J (1958) Psychotherapy by reciprocal inhibition, Stanford University Press, Stanford CA. 